\title{
Hybrid Neural Approach for Face and Associated Emotion Recognition Using Swarm Intelligence-Based Ensemble Network
}

\author{
Mrs. Ismath Unnisa ${ }^{1}$ \\ Research Scholar, Computer Science \& Engineering Department \\ HKBK College of Engineering \\ Bangalore, Karnataka 560 045, India \\ ismath.ayaan@gmail.com \\ http://www.hkbk.edu.in \\ Dr. Loganathan. $\mathrm{R}^{2}$ \\ Professor \& Head of Computer Science \& Engineering Department \\ HKBK College of Engineering \\ Bangalore, Karnataka 560 045, India \\ drloganathanr@gmail.com \\ http://www.hkbk.edu.in
}

\begin{abstract}
In this work, a hybrid approach which carries the Radial Basis Function Neural Network and Multilayer Perceptron Neural Network have been applied in a cascaded manner to recognize the face and associated emotions. The variability of individual classifier performances has been reduced by providing the ensemble approach. The formation of ensemble has been developed using the intelligent manner with the help of particle swarm optimization. The applied ensemble approach provided the weighted importance of individual entities according to their performances. The proposed ensemble approach has been proven to be useful over the development of ensemble classifier for XOR classification problem. Each face has been carried with a different form of emotion which has been tested and performance was compared against the individual classifier module.
\end{abstract}

Keywords: Face Recognition; Emotion Recognition; Neural Network; Radial Basis Function; Multilayer Perceptron; Ensemble and Particle Swarm Optimization.

\section{Introduction}

A human performs a task of face recognition continuously as well as effortlessly everyday as a routine. It includes human-computer interaction, biometric authentication, multimedia management and surveillance. As there is huge availability of desktops that are powerful and low cost as the embedded computing systems, this has led to a focus on automatic processing of digital images and videos in applications. The research and development is automated for face recognition. The basic challenges are not only the face recognition but also numerous practical applications which require human identification. One of the primary biometric technologies is face recognition and it has gained more importance owing to fast advancing technologies like internet, mobile devices and digital cameras and this has increased demands on security. Over the other biometric technologies, the face recognition has many advantages. It is non-intrusive, natural as well as easy to use. The highest compatibility in Machine Readable Travel Documents (MRTD) is scored by the facial features among the six biometric attributes depending on number of evaluation factors like machine requirements, enrolment renewal, public perception and the face recognition system, which identifies the faces automatically from the images and videos. There are two modes in which it can operate: (1) Face verification/authentication (2) Face identification/recognition. Face verification includes one-to-one match which will compare query face image and template face image whereas face identification includes one-to-many matches which compares query face image with all template face images present in database to find out the query face identity. Watch-list check is one of the face recognition scenarios. Here, the query face is matched with a list of suspects that is one-to-few matches. In human communication, the important factors are facial emotions which help in understanding the intentions of others. So, in general, other people's emotional states like sadness, joy, anger is inferred by using vocal tone and facial expression. Based on 
various surveys, one-third of human communication is conveyed by verbal components, whereas two-thirds is conveyed by nonverbal components. Among various nonverbal components, by considering emotional meaning in interpersonal communications, one of the main information channels is facial expressions. Thus, it is obvious that facial emotion research has gained attention with applications not only in cognitive and perceptual sciences, but also in computer animations and affective computing. Automatic facial emotion recognition (FER) has rapidly increased in recent times with development of artificial intelligent techniques. Facial expressions play important role in interpersonal relations. For natural human-machine interfaces, automatic recognition of facial expressions is an important component. It can also be used in clinical practice and also in behavioral science. Even though without any effort or delay, humans can recognize facial expressions but still for a machine, it is a challenge to recognize reliable facial expressions.

\section{Literature Survey}

On surveying the methods and systems present in paper [1], we found that the automatic facial expression analysis is being used. Distributed fingerprint classification rule (SFCA) has overcome the drawback of free face recognition in [2]. In [3], the technique is planned in a way that it recognizes the faces acknowledged by us when there are only few or perhaps only one face. These examples used to be corrupted by the annoying variables with the linear or nonlinear method. This survey presents a way for facial expression recognition using cascade of neural networks in paper [4]. An input of an algorithm receives image of a page, which is normal and results with an emotion that the face expresses. Testing was done for one- and multi-layered networks in order to determine the best classifier for recognizing the particular emotion. In research fields of social psychology and social neuroscience, especially with respect to neuropeptide oxytocin, study of facial expressions of emotions has become essential. In paper [5], it has been examined how processing of emotions in faces has been influenced by oxytocin and by reviewing the intranasal administration studies of selective attention, automatic processing and emotion recognition by using the combination of Convolutional Neural Network and specific image preprocessing steps, facial expression recognition can be done [6]. A detailed overview on FER including convolutional network and deep learning has been discussed earlier in paper [7]. To deal with the facial emotion recognition problem, a framework has been proposed in paper [8]. Facial muscle deformations, gradient features are unusually sensitive to the object deformation results in facial expressions, hence to encode the facial components, gradients were applied as featured in paper [9]; to command a mobile robot (pionner-3DX), a facial expression recognition system is used. In classification, six facial emotion classes have presented based on the single Deep Convolutional Neural Networks (DNNs) and it contains deep residual blocks in [10] and convolutional layers to analyze images and identified face $\mathrm{CNN}$ model can be trained using Convolutional Neural Networks. In [11], create system has been proposed to recognize the student's emotion from their faces. There are 3 steps: Using $\mathrm{CNN}$ detection of face, using Haar cascades, normalization and emotion recognition. Facial landmark and HOG are pre-processing incorporated in CNN in [12] in order to achieve a better accuracy over the facial recognition. Discrimination between the complimentary emotion and dominant emotion is usually weak due to which it creates more problems in compound facial emotion recognition. In this, 2-stage recognition methods are used in [13]. Coarse recognition stage is the first stage and fine recognition is the second stage, which is used for enhancing the classification for symmetrical emotion labels. For feature extraction and pre-processing phases, [14] utilizes a novel graph-based binary pattern, which is Quintet Triple Binary Pattern (QTBP) and Discrete Wavelet Transform (DWT). Meanwhile, for the task of classification, Support Vector Machine (SVM) and K-Nearest Neighbours (KNN) were employed. To recognize the facial emotions from virtual, stylized and human characters, multi-block Deep Convolutional Neural Networks (DCNN) model is applied in [15]. For facial expression recognition, [16] has done the comparative performance between the traditional machine learning approaches and deep learning models. In order to evaluate the consistency between subjective feeling of emotions and their spontaneous facial displays and to compare the recognition of the type of displays from automatic facial expression by human perceivers, classifier, the detail experiments are utilized as noted in paper[17].

\section{Proposed Method for Face \& Facial Recognition}

The general construction of automated system for face and associated emotion recognition is shown in the Fig.1. The Fig. 1 is divided into two halves. The upper half describes the general approach as to how the work is carried out and the lower half depicts the applied corresponding part in this work to show how the corresponding position function works. The image shaping is applied to ensure the same size is used for all input images so that without any variation in all images, it could process with the same module; the gray scale conversion of image is applied because color of skin is not considered in this work as a feature trait. Principle Component Analysis (PCA) is applied to extract the core features of the image by making the uncorrelated information as dimensionality reduction has been achieved by considering the few components of PCA outcomes. Radial Basis Function Neural 
Network (RBFNN) is considered as the classifier module for the face recognition, which carries the adaptive form of architecture in terms of adaptive kernel function, which has been considered in the hidden nodes. The adaptive form has been applied to make the Gaussian function mean and spread parameters as discussed in paper [18]. In the recognition of the facial expression, an adaptive form of MLP architecture is applied which carries the adaptive slope of the transfer function available in the active nodes. This adaptive slope provides one extra dimension of freedom to learn the things in better manner. The details are given in paper [19]. The distance measure is applied in the knowledge domain with help of correlation-based outcomes between the input features and the trained neural network parameters. In the proposed method, there is no need to store the image templates, which makes the process of execution faster.

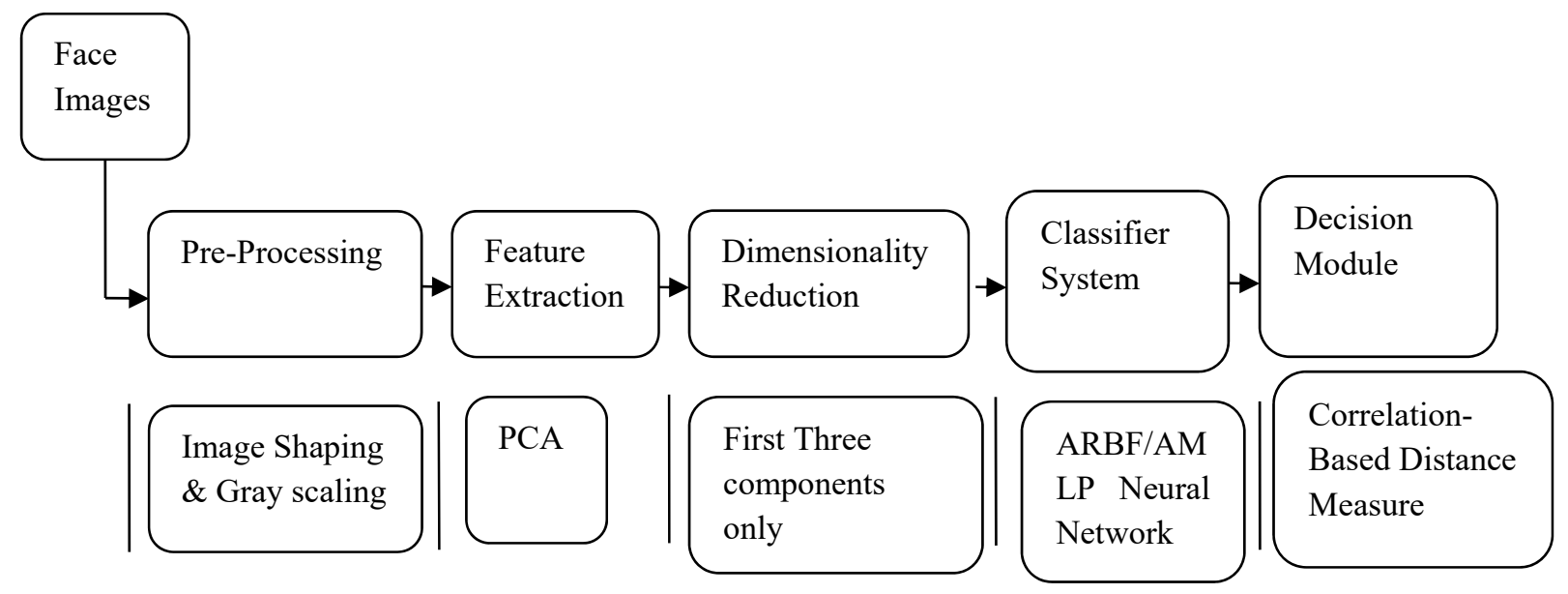

Fig.1. Abstract modular diagram applied in proposed face and associated expression recognition.

The applied form of proposed ensemble approach is shown in Fig.2. There are two ensemble parts under this approach. The first ensemble is created for the face recognition with the help of ARBF. The three ARBF have been trained and their outcomes are combined through dynamic weighted form of PSO (DyPSO) to define the final outcomes. The decision module 1 is used to recognize the face under minimum correlation position value. Once the face has been recognized, it triggers the emotion knowledge bank which carries the trained weights for different types of expression. Over the possibilities of all emotions, an ensemble of AMLP through DyPSO defines the available emotion at present with the current face by finding the minimum distance position in correlation domain with the help of decision module 2 as shown in Fig.2. In the domain of swarm intelligence, Particle Swarm Optimization (PSO) appears as one of the finest approaches which carries the simplicity in design and has the capability to achieve a good solution with faster convergence rate. There are three types of populations updated at every iteration. The first is velocity population in which each individual member is updated as the function of inspirational form having the references with respect to best solution and self-motivation as shown in Eq.1. The second population is solution population in which each individual member is updated by adding correspondingly new velocity vector as shown in Eq.2. The third population is self-best population where the individual members update their result that was best achieved in the past.

$$
\begin{gathered}
V_{i}^{(k+1)}=\chi\left[w V_{i}^{k}+c_{1} \text { rand }_{1} \times\left(\text { pbest }_{i}-s_{i}^{k}\right)+c_{2} \text { rand }_{2} \times\left(\text { gbest }_{i}-s_{i}^{k}\right)\right] \\
S_{i}^{(k+1)}=S_{i}^{k}+V_{i}^{k+1}
\end{gathered}
$$

Where ' $i$ ' and ' $k$ ' carry the member number and iteration number, $V_{i}^{k}$ represents the velocity vector, $\mathrm{W}$ represents the inertia weight to control the amplitude of change, while $C_{1} \& C_{2}$ are social and cognitive constants. $S_{i}^{k}$ represents present position of a solution, pbest $_{i}$ represents achieved best in the past, gbest $_{i}$ is the best solution available in population. $\chi$ is another constant factor to ensure the convergence and called as constriction factor. In the proposed solution, the inertia weight is considered to be dynamic so that with each iteration, there will be a change and this change is based on the logic to show that progress has taken place and solutions are moving towards the optimal solution region. Hence the search steps become smaller with each iteration as shown in Eq 3. 


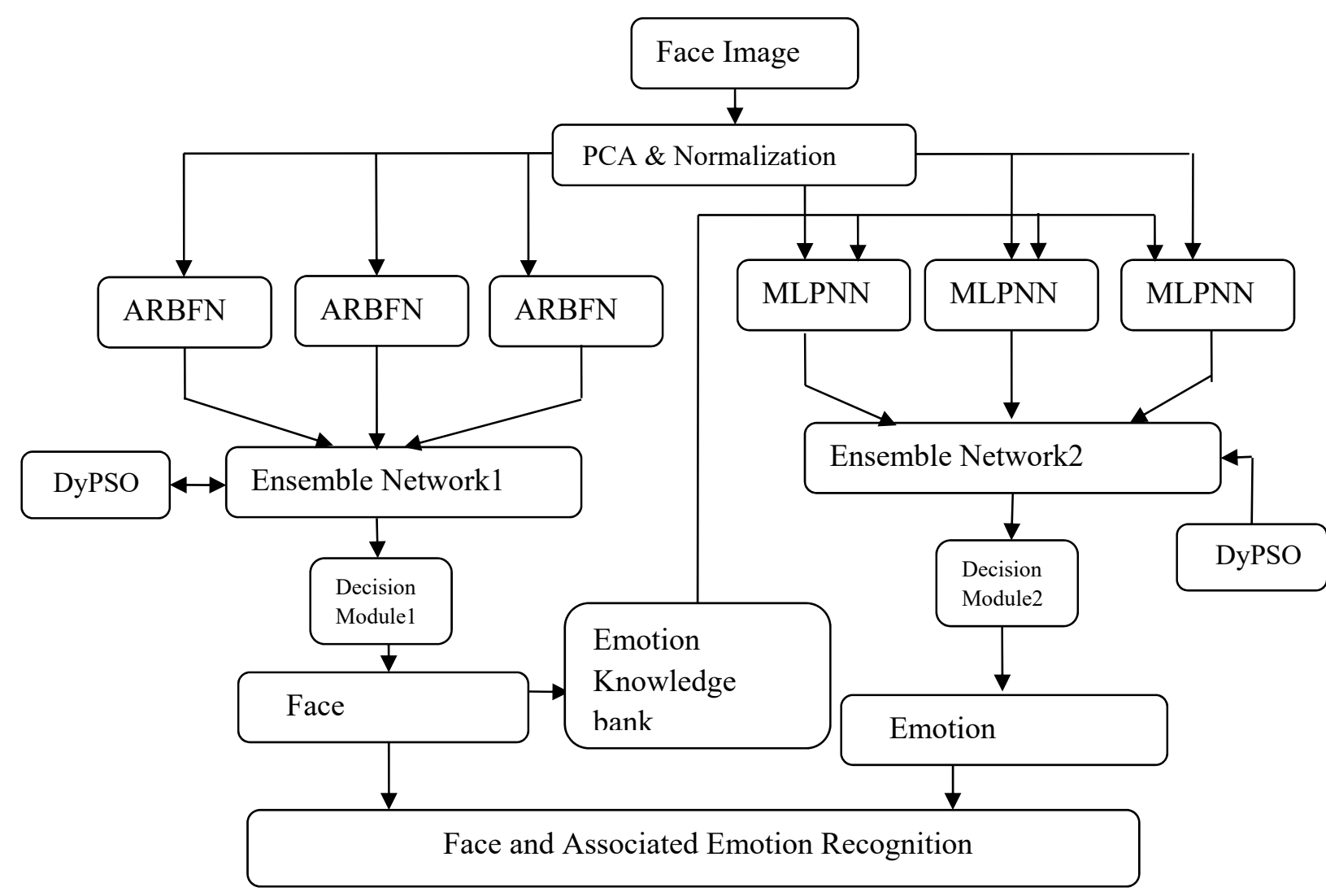

Fig. 2: Proposed Ensemble Approach to Recognize the Face and Facial Expression

$$
w=w_{\max } \frac{\left(w_{\max }-w_{\min }\right)}{\text { iter }_{\max }} \times i t e r
$$

Where $w_{\max }$ : starting maximal initial weight; $w_{\min }:$ minimum final weight; $i t e r_{\max }:$ number of maximum iteration; iter: represents present iteration number.

The DyPSO is applied to form the ensemble network as shown in Fig.3. There are three different classifier entities in the ensemble network whose numeric outcome's output becomes the input to the neural ensemble. Hence, there is a need to search the three parameters w1, w2, and w3 corresponding to each connection as shown in Fig.3. The multiplied outcomes along with classifier outcomes corresponding to their weight value are summed and transformed by sigmoid function to deliver the final outcome. The error is estimated with target and minimization of error is achieved through search process of PSO. 


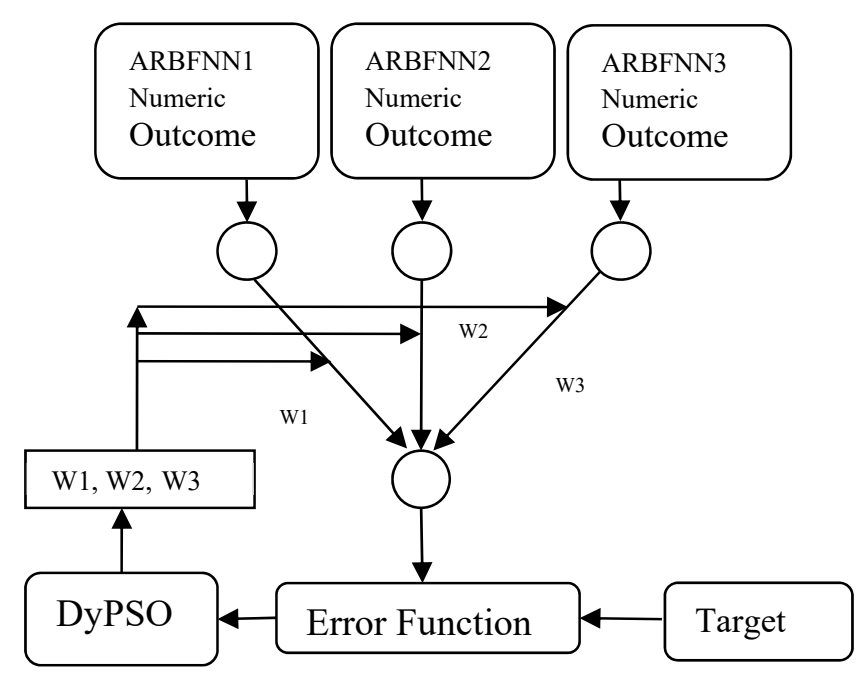

Fig.3 Formation of Ensemble Network using DyPSO

The development of decision module is based on the correlation between the inputs and trained values of the network parameters which is shown in Fig.4. First through the training cases, correlation values are estimated and stored. At the time of test for a particular test image, the different network parameters are estimated with the correlation value through the network architecture and forms the test correlation set value. The training and test correlation values are compared against each other and minimum value position is considered as defined identification of test input image. 


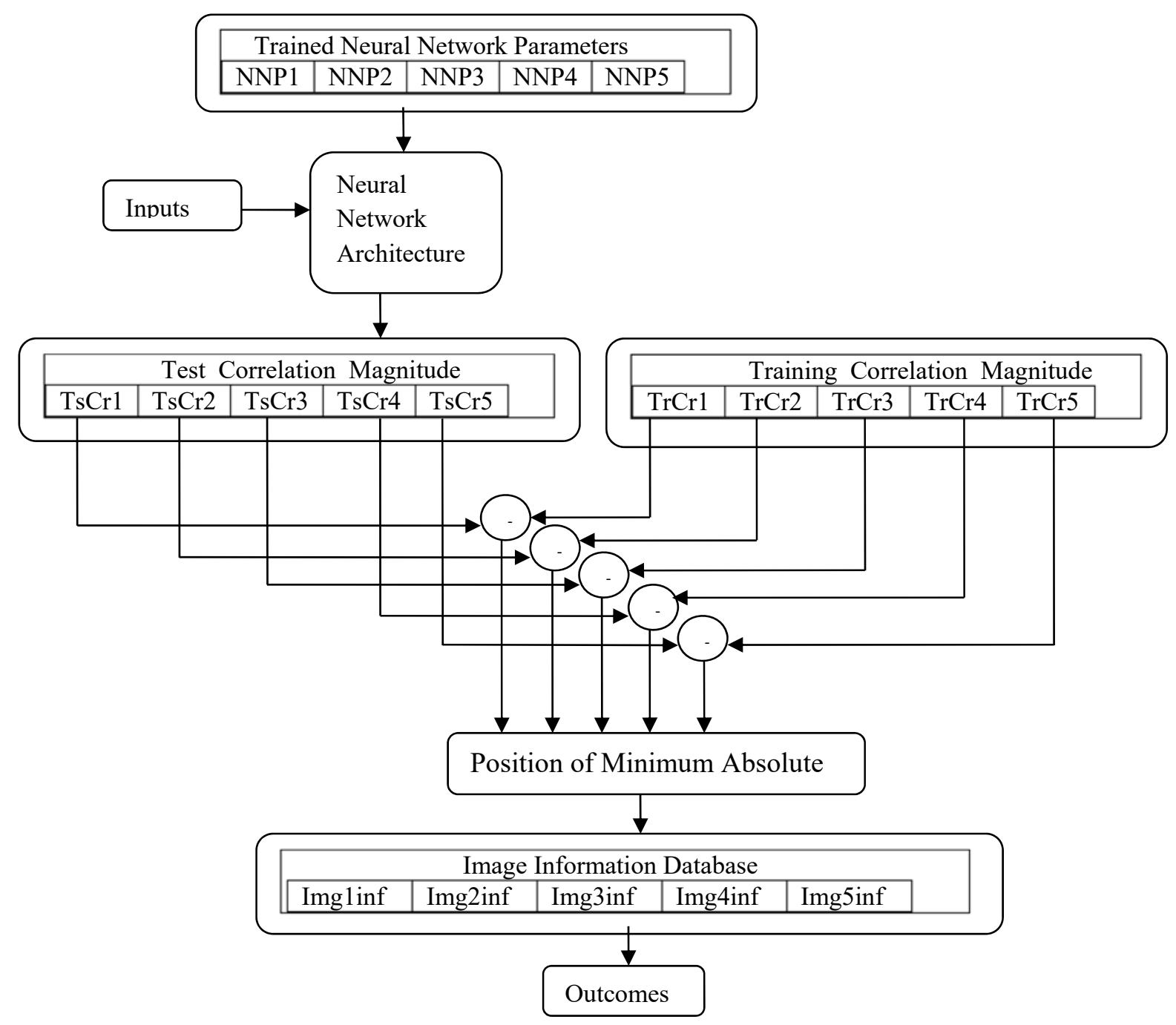

Fig.4. Correlation Distance-Based Distance Measure

\section{Experimental Results}

To understand the benefit of proposed form of ensemble, a benchmark problem of XOR classification has been considered using the ARBF, which carries the architecture size as [ [ 231 1]. The three ARBF has been considered to form an ensemble network. The learning is applied for 500 iterations and obtained convergence as Sum of the Absolute Error (SAE) as shown in Fig.5. It is observed that even with same environment, there appear different patterns of convergence. The obtained result of network parameters for all the three classifiers and the ensemble modules are shown in Table 1. The final outcome for the different inputs of XOR problem with individual and ensemble approach are shown in Table 2. It can be observed that there were significant variations among the individual ARBF classifier performances and comparatively large errors in outcomes against ensemble part. 


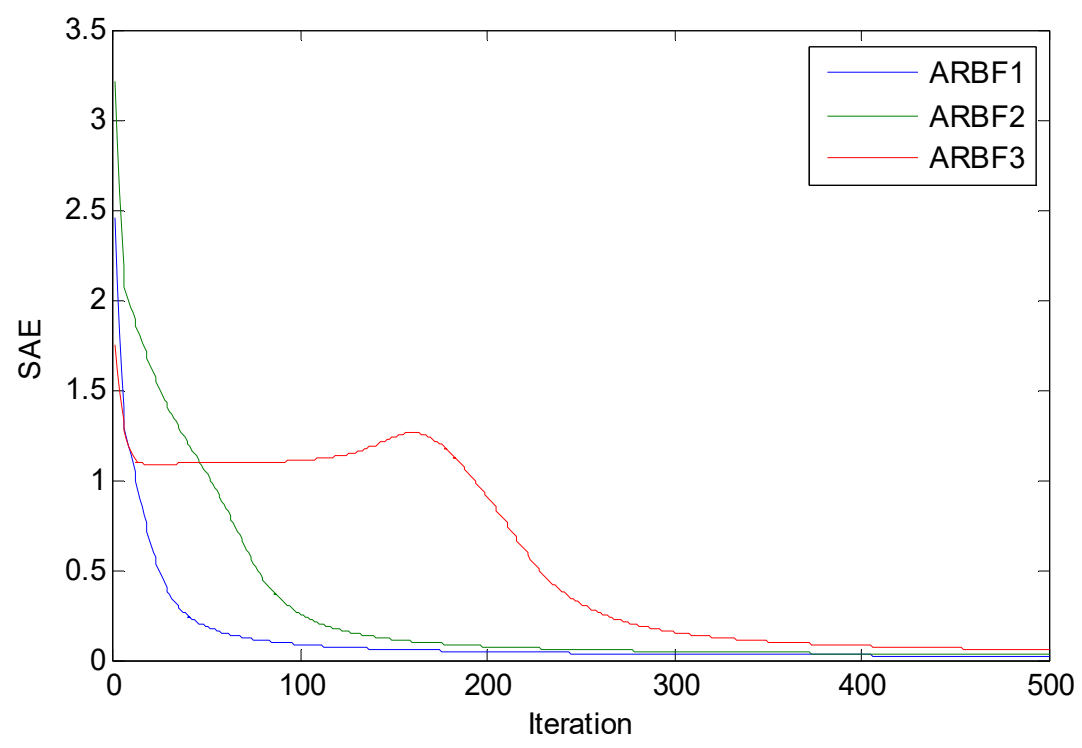

Fig.5 Convergence characteristics for different ARBF for XOR problem

\begin{tabular}{|c|c|c|c|}
\hline \multirow{2}{*}{$\begin{array}{l}\text { Network } \\
\text { parameter }\end{array}$} & ARBF1 & ARBF2 & ARBF3 \\
\hline & $1^{\text {st }} \mathrm{hn} \quad 2^{\text {nd }} \mathrm{hn}$ & $1^{\text {st }} \mathrm{hn} \quad 2^{\text {nd }} \mathrm{hn} \quad 3^{\text {rd }} \mathrm{hn}$ & $2^{\text {nd }} \mathrm{hn} \quad 3^{\text {rd }} \mathrm{hn}$ \\
\hline $\begin{array}{l}\text { Kernel } \\
\text { mean }\end{array}$ & $\begin{array}{rrr}-0.0721 & -0.1850 & 0.9799 \\
-0.0683 & 0.9709 & -0.1768\end{array}$ & $\begin{array}{ccc}-0.1609 & -0.1115 & 0.8379 \\
-0.0733 & 1.0058 & -0.2469\end{array}$ & $\begin{array}{lll}0.3800 & 1.0505 & 1.0942 \\
1.2464 & 0.0037 & 1.0226\end{array}$ \\
\hline $\begin{array}{l}\text { Kernel } \\
\text { spread }\end{array}$ & $\begin{array}{lll}0.6780 & 0.5472 & 0.5392\end{array}$ & $\begin{array}{lll}0.5721 & 0.4629 & 0.6254\end{array}$ & $0.4295 \quad 0.4159$ \\
\hline $\begin{array}{l}\text { Output } \\
\text { weight }\end{array}$ & $\begin{array}{lll}-0.0828 & 1.1305 & 1.1211\end{array}$ & $\begin{array}{lll}-0.2072 & 1.0613 & 1.2513\end{array}$ & $0.9885-0.6406$ \\
\hline $\begin{array}{l}\text { Ensemble } \\
\text { Weight } \\
(1.0 \mathrm{e}+03 *)\end{array}$ & 1.8144 & -1.6439 & -0.1605 \\
\hline
\end{tabular}

Table1: Individual network parameters after training by different classifier

\begin{tabular}{|l|l|l|l|l|}
\hline Inputs & ARBF1 & ARBF2 & ARBF3 & PSOEN \\
\hline$\left[\begin{array}{ll}0 & 0\end{array}\right]$ & -0.0007 & -0.0016 & 0.0451 & $\mathbf{0 . 0 0 2 6}$ \\
\hline$\left[\begin{array}{ll}0 & 1\end{array}\right]$ & 0.9991 & 0.9995 & 0.9956 & $\mathbf{0 . 9 9 9 9}$ \\
\hline$\left[\begin{array}{ll}1 & 0\end{array}\right]$ & 0.9992 & 0.9978 & 0.9991 & $\mathbf{1 . 0 0 0 0}$ \\
\hline$\left[\begin{array}{ll}1 & 1\end{array}\right]$ & 0.0194 & 0.0252 & -0.0021 & $\mathbf{0 . 0 0 2 6}$ \\
\hline
\end{tabular}

Table2. Comparative Outcomes by Individual Classifier and PSO-based Ensemble

To obtain the experiment over face and facial expression recognition, images from Yale Face Database [20] which has 15 images with 6 different form of expressions is considered as shown in Appendix. The whole experiment is divided into two parts:

1) In the training phase of face recognition, only normal state of each image is considered while in the test phase, different facial states of individual images is considered and the purpose is to recognize the face.

2) In the second phase, any image carrying the anomalies has been considered and the purpose is to recognize the face as well as facial expression carried by the face.

\subsection{Case 1: Sad and Happy Face with Gaussian Noise}

Image ID 3 for the face recognition: The two different states of face "sad" and "happy" are considered while image is corrupted with different forms of noise. The outcomes are shown in terms of normalized correlation confidence (NCC) by all the individual classifiers as well as by the ensemble approach. It is clear that all the 
three individual classifiers are able to recognize the face by having the maximum NCC value at the position of 3, but it can also be observed that Ensemble-Based Approach (PSOEN) has carried the low value of NCC for other faces when compared to the individual classifiers. This shows that there is more robustness in the outcomes as shown in Fig. 6 and Fig. 7.

\subsubsection{Sad Face with Gaussian Noise}
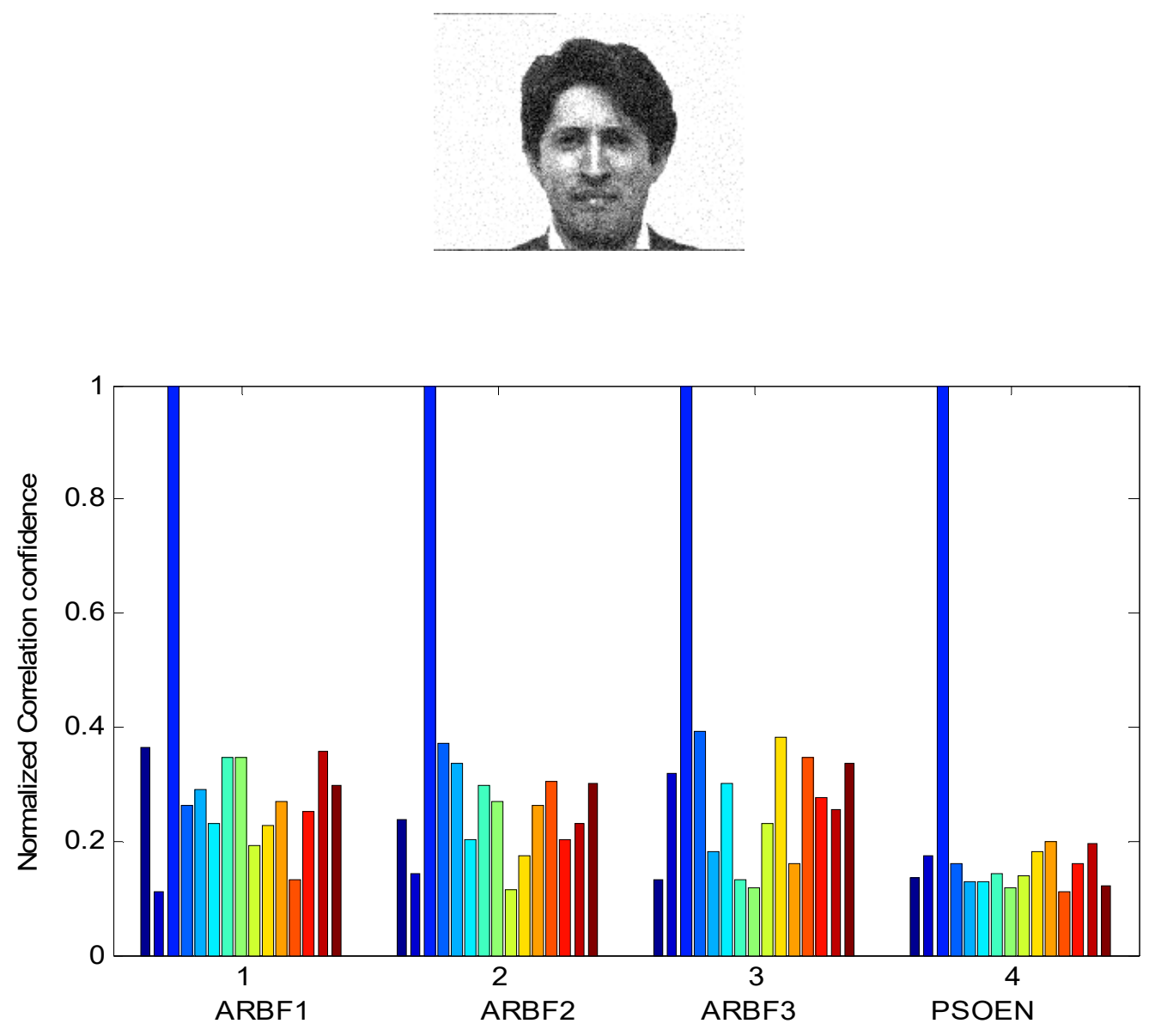

Fig6. Face recognition NCC bar plot for the image id 3 for sad face

\subsubsection{Happy face with Gaussian noise}

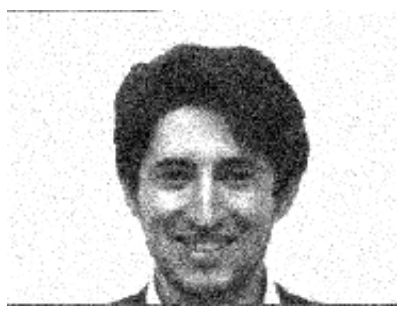




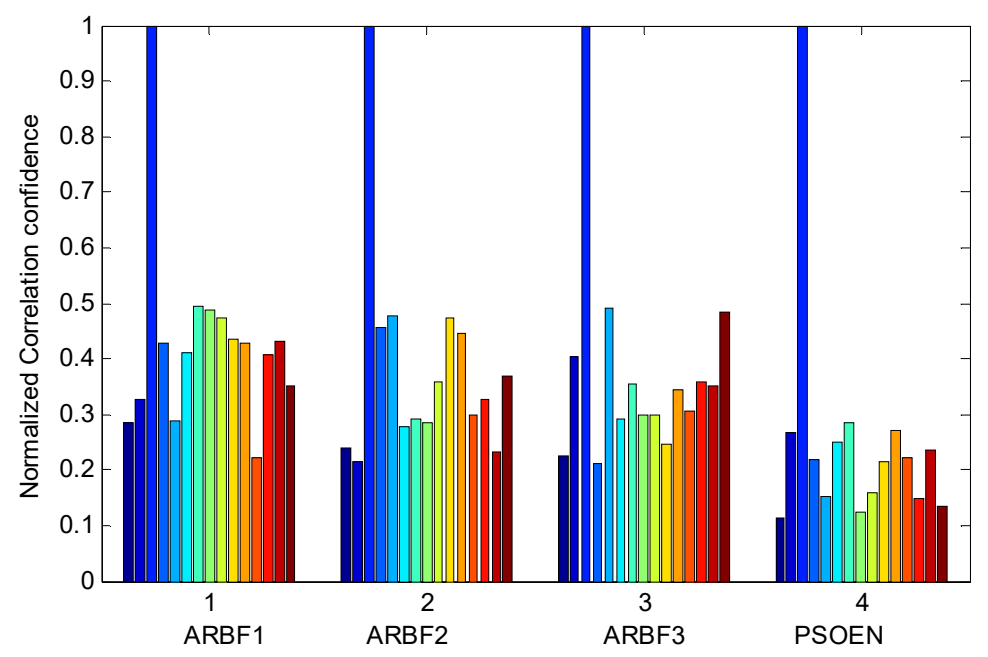

Fig7. Face Recognition NCC Bar Plot for the Image ID 3 for Happy Face

\subsection{Case 2: Happy Face with Gaussian and Speckle Noise}

In case 2, the test input face is carried for image ID 11 from the database over which training was not given for face recognition. The face image is heavily corrupted with Gaussian and speckle noise and carries the happy state of facial expression as shown below. The problem is to recognize the face and associated facial expression.

\subsubsection{Happy Face with Gaussian and Speckle Noise}

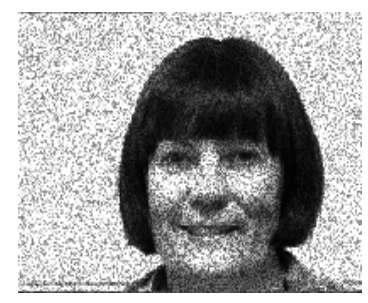

The outcome of NCC value for the face recognition of image ID 11 is shown in Table 3 and we also observe that all individual classifiers have the maximum NCC value at the position of image ID 11. The ensemble has once again shown the lower value of NCC for other IDs when compared to the individual classifier as shown in Fig.8.

\begin{tabular}{|l|l|l|l|l|l|l|l|l|l|l|l|l|l|l|l|l|}
\hline & \multicolumn{10}{|c|}{ Image ID } \\
\hline & 1 & 2 & 3 & 4 & 5 & 6 & 7 & 8 & 9 & 10 & 11 & 12 & 13 & 14 & 15 \\
\hline & & & & & & & & & & & & & & & \\
\hline AR & 0.2 & 0.2 & 0.1 & 0.1 & 0.3 & 0.1 & 0.2 & 0.1 & 0.3 & 0.1 & 1.0 & 0.3 & 0.2 & 0.1 & 0.2 \\
BF1 & 057 & 543 & 746 & 434 & 879 & 298 & 931 & 800 & 934 & 390 & 000 & 069 & 146 & 713 & 278 \\
& & & & & & & & & & & & & & & \\
\hline AR & 0.1 & 0.2 & 0.2 & 0.1 & 0.1 & 0.3 & 0.3 & 0.3 & 0.1 & 0.2 & 1.0 & 0.3 & 0.3 & 0.2 & 0.3 \\
BF2 & 675 & 539 & 566 & 216 & 248 & 012 & 518 & 499 & 241 & 756 & 000 & 384 & 222 & 853 & 709 \\
& & & & & & & & & & & & & & & \\
\hline AR & 0.2 & 0.1 & 0.3 & 0.2 & 0.2 & 0.2 & 0.2 & 0.1 & 0.3 & 0.2 & 1.0 & 0.2 & 0.2 & 0.2 & 0.2 \\
BF3 & 818 & 510 & 002 & 945 & 376 & 908 & 828 & 444 & 361 & 628 & 000 & 012 & 501 & 212 & 769 \\
& & & & & & & & & & & & & & & \\
\hline PSO & $\mathbf{0 . 1}$ & $\mathbf{0 . 1}$ & $\mathbf{0 . 1}$ & $\mathbf{0 . 1}$ & $\mathbf{0 . 1}$ & $\mathbf{0 . 1}$ & $\mathbf{0 . 1}$ & $\mathbf{0 . 1}$ & $\mathbf{0 . 1}$ & $\mathbf{0 . 1}$ & $\mathbf{1 . 0}$ & $\mathbf{0 . 1}$ & $\mathbf{0 . 1}$ & $\mathbf{0 . 1}$ & $\mathbf{0 . 1}$ \\
EN & $\mathbf{1 8 3}$ & $\mathbf{3 0 5}$ & $\mathbf{9 7 9}$ & $\mathbf{9 5 4}$ & $\mathbf{4 6 6}$ & $\mathbf{1 5 9}$ & $\mathbf{7 6 6}$ & $\mathbf{0 6 4}$ & $\mathbf{1 0 2}$ & $\mathbf{3 1 9}$ & $\mathbf{0 0 0}$ & $\mathbf{3 8 7}$ & $\mathbf{2 9 3}$ & $\mathbf{0 6 7}$ & $\mathbf{3 1 0}$ \\
\hline
\end{tabular}




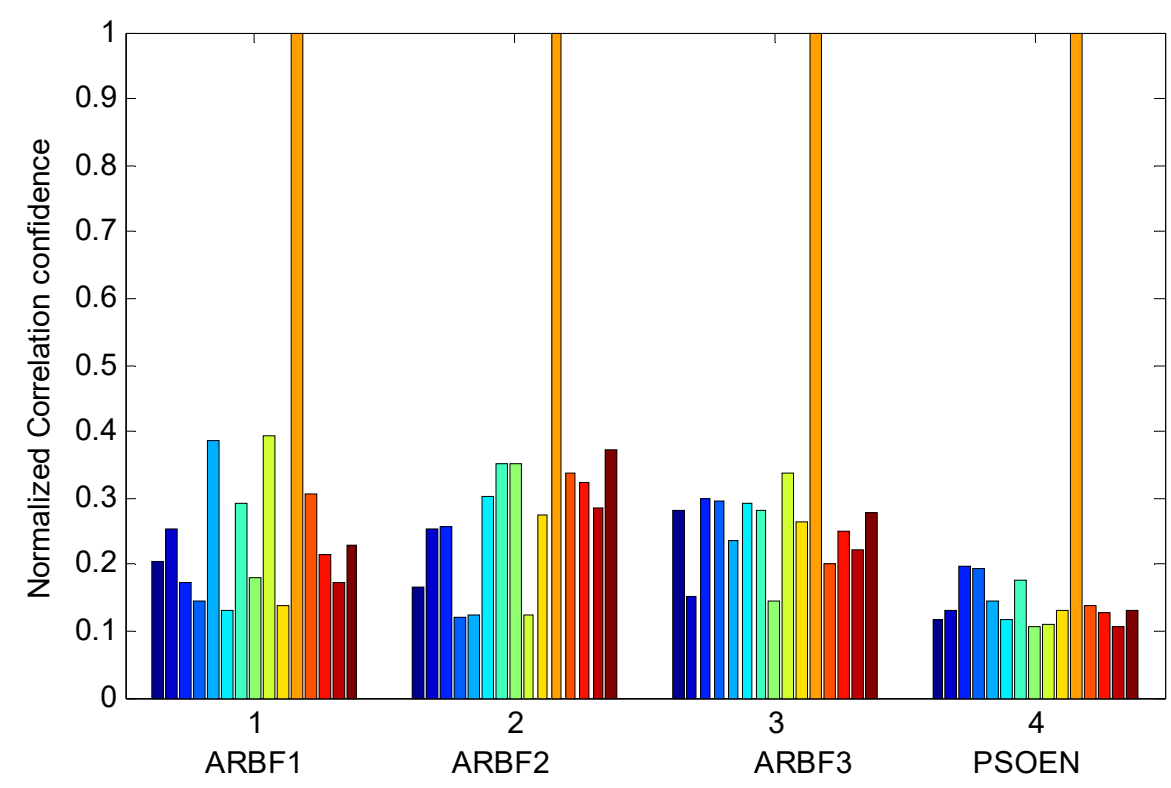

Fig8. Face recognition NCC bar plot for the image id 11 for happy face

The conventional approach of forming the ensemble is to take the mean-based approach, where the final value of outcome is derived from the mean of all and here, we call it as MEN. The comparative values between MEN and PSOEN are shown in Table 4 and in Fig. 9 and it is observed that PSOEN are resulted with much lower values to other ID places except ID 11. This shows the robustness of the proposed ensemble. The facial expression outcome is shown in Table 5 where maximum NCC value at position 1 ensures about the state of happiness by all the classifiers. It is also observed that PSOEN is given a low value of NCC over other places when compared to individual classifiers.

\begin{tabular}{|c|c|c|c|c|c|c|c|c|c|c|c|c|c|c|c|}
\hline & \multicolumn{15}{|c|}{ Image ID } \\
\hline & 1 & 2 & 3 & 4 & 5 & 6 & 7 & 8 & 9 & 10 & 11 & 12 & 13 & 14 & 15 \\
\hline MEN & $\begin{array}{l}0.21 \\
83\end{array}$ & $\begin{array}{l}0.21 \\
97\end{array}$ & $\begin{array}{l}0.24 \\
38\end{array}$ & $\begin{array}{l}0.18 \\
65\end{array}$ & $\begin{array}{c}0.25 \\
01\end{array}$ & $\begin{array}{l}0.24 \\
06\end{array}$ & $\begin{array}{l}0.30 \\
92\end{array}$ & $\begin{array}{l}0.22 \\
48\end{array}$ & $\begin{array}{l}0.28 \\
45\end{array}$ & $\begin{array}{l}0.22 \\
58\end{array}$ & $\begin{array}{l}1.00 \\
00\end{array}$ & $\begin{array}{l}0.28 \\
22\end{array}$ & $\begin{array}{l}0.26 \\
23\end{array}$ & $\begin{array}{l}0.22 \\
59\end{array}$ & $\begin{array}{l}0.29 \\
19\end{array}$ \\
\hline $\begin{array}{l}\text { PSOE } \\
\mathrm{N}\end{array}$ & $\begin{array}{l}0.11 \\
83\end{array}$ & $\begin{array}{l}0.13 \\
05\end{array}$ & $\begin{array}{l}0.19 \\
79\end{array}$ & $\begin{array}{l}0.19 \\
54\end{array}$ & $\begin{array}{l}0.14 \\
66\end{array}$ & $\begin{array}{l}0.11 \\
59\end{array}$ & $\begin{array}{l}0.17 \\
66\end{array}$ & $\begin{array}{l}0.10 \\
64\end{array}$ & $\begin{array}{l}0.11 \\
02\end{array}$ & $\begin{array}{l}0.13 \\
19\end{array}$ & $\begin{array}{l}1.00 \\
00\end{array}$ & $\begin{array}{l}0.13 \\
87\end{array}$ & $\begin{array}{l}0.12 \\
93\end{array}$ & $\begin{array}{l}0.10 \\
67\end{array}$ & $\begin{array}{l}0.13 \\
10\end{array}$ \\
\hline
\end{tabular}

Table 4. Comparison of NCC values by MEN and PSOEN

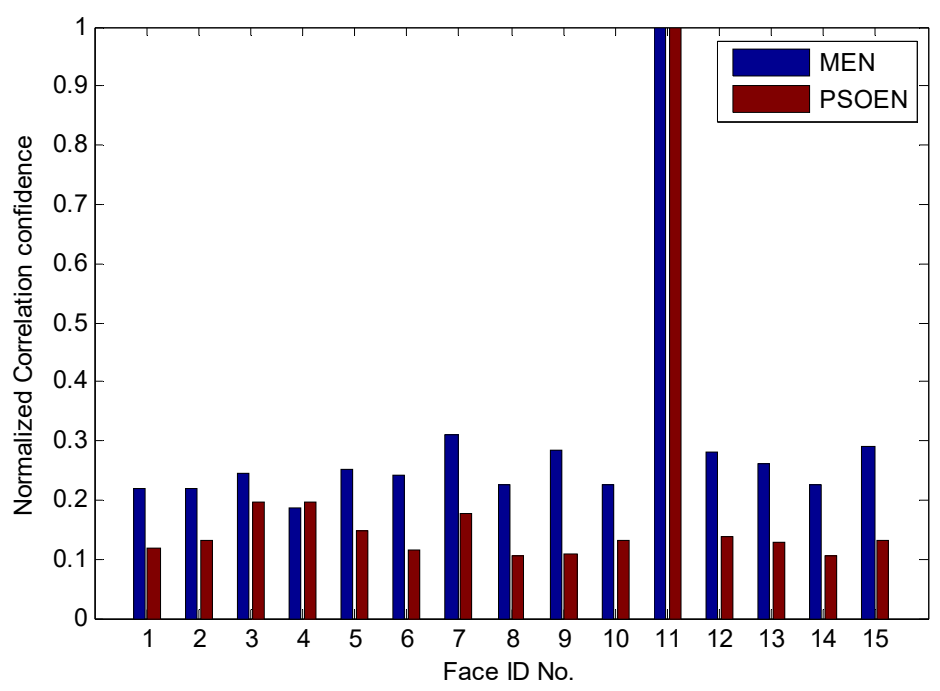

Fig.9 NCC value comparison between MEN and PSOEN 


\begin{tabular}{|l|l|l|l|l|l|l|}
\hline & \multicolumn{7}{|c|}{ Facial expression ID } & \multicolumn{1}{c|}{6} \\
\hline & 1 & 2 & 3 & 4 & 5 & 0.3686 \\
\hline ARBF1 & 1.0000 & 0.5769 & 0.2379 & 0.5896 & 0.4844 & \\
\hline ARBF2 & 1.0000 & 0.3902 & 0.2270 & 0.6670 & 0.4347 & 0.2811 \\
\hline ARBF3 & 1.0000 & 0.4839 & 0.4654 & 0.2650 & 0.2060 & 0.5971 \\
\hline PSOEN & $\mathbf{1 . 0 0 0 0}$ & $\mathbf{0 . 3 0 5 7}$ & $\mathbf{0 . 2 3 3 1}$ & $\mathbf{0 . 3 2 0 4}$ & $\mathbf{0 . 2 5 2 6}$ & $\mathbf{0 . 3 3 0 8}$ \\
\hline
\end{tabular}

Table.5 Facial Expression NCC Values over Different Positions

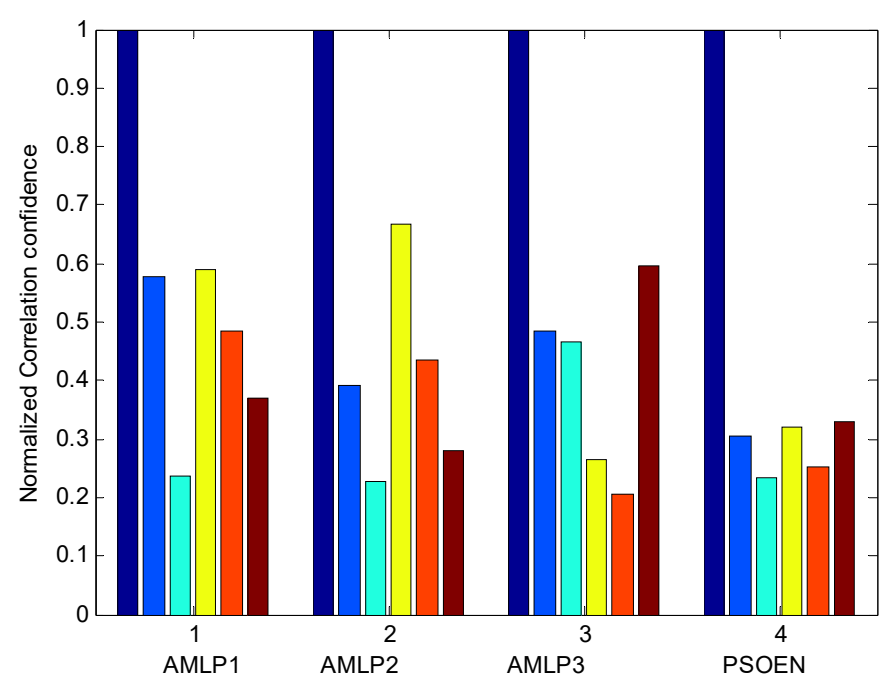

Fig10. Facial Expression NCC Bar Plot for the image ID 11

The obtained NCC values for facial expressions are represented as bar plot shown in Fig. 10 to observe the clarity. The benefit of PSOEN against MEN for facial recognition over the given input is shown in Fig. 11 and observed that PSOEN has given much lower value to other expressions.

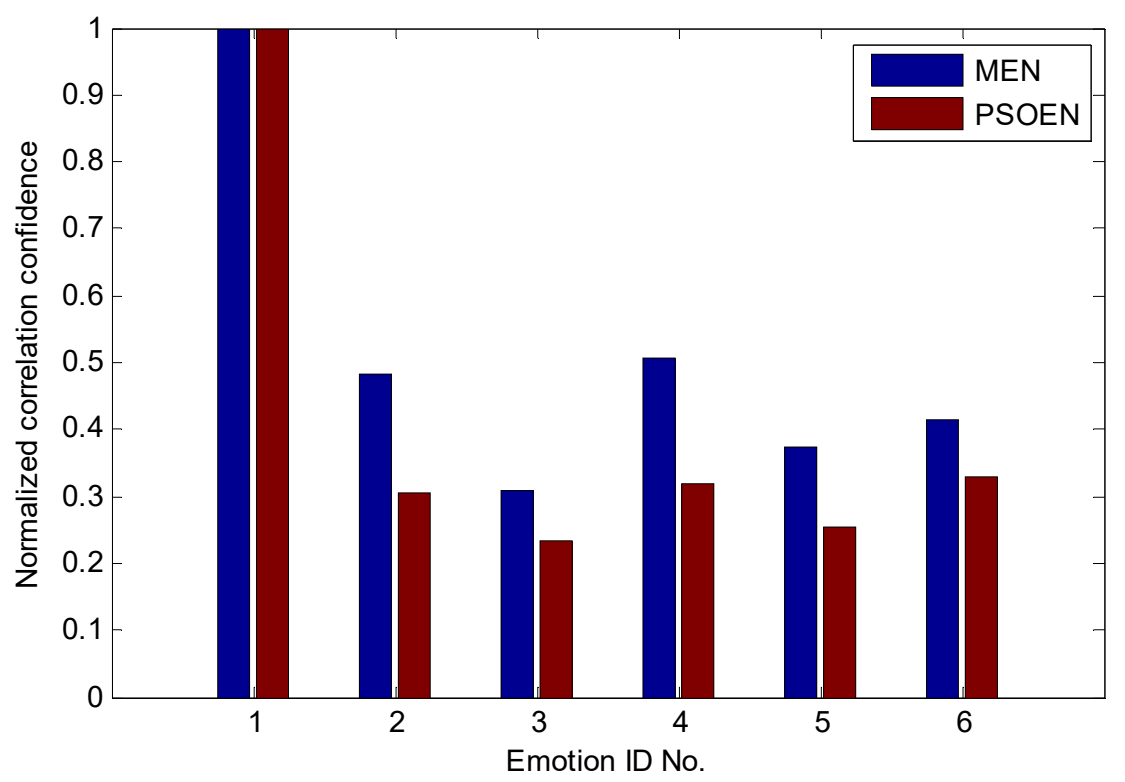

Fig. 11 Facial Expression NCC Value Comparison between MEN and PSOEN 


\section{Conclusion}

The proposed work is to focus on determining a robust means to solve the problem of outcome variability of the individual classifier performances. This problem has been resolved by proposing an Evolved Neural Ensemble Network using the PSO. The simplicity of PSO and effectiveness has delivered the possibilities to obtain the compact and robust ensemble, which has the capability to stabilize the output along with an increase in the quality of decision. The proposed ensemble has been applied to recognize the face and associated expression efficiently. The test images are carried with lots of vulnerability when compared to the original images. The proposed ensemble is very effective when compared to conventional approach of ensemble network by considering the mean value function. The proposed method is simple in implementation, efficiency and generalized in nature.

\section{References}

[1] B.Fasel JuergenLuettin, “Automatic Facial Expression Analysis: A Survey,” Recognition, Volume, January 2003 , Pages $259-275$.

[2] Tomas Larrain; John S. Bernhard; Domingo Mery; Kevin W. Bowyer, "Face Recognition Using Sparse Fingerprint Classification Algorithm" IEEE Transactions on Information Forensics and Security (Volume: 12, Issue: 7), 2017.

[3] Yuan Gao,Jiayi Ma, Alan L. Yuille, "Semi-Supervised Sparse Representation-Based Classification for Face Recognition With Insufficient Labeled Samples” IEEE Transactions on Image Processing (Volume: 26, Issue: 5), 2017.

[4] Kukla E., Nowak P. (2015) Facial Emotion Recognition Based on Cascade of Neural Networks. In: Zgrzywa A., Choroś K., Siemiński A. (eds) New Research in Multimedia and Internet Systems. Advances in Intelligent Systems and Computing, Vol 314. Springer, Cham

[5] Ellenbogen M.A. (2017) Oxytocin and Facial Emotion Recognition. In: Hurlemann R., Grinevich V. (eds) Behavioral Pharmacology of Neuropeptides: Oxytocin. Current Topics in Behavioral Neurosciences, Vol 35. Springer, Cham

[6] André TeixeiraLopes, Alberto F.DeSouza,'Facial Expression Recognition with Convolutional Neural Networks: Coping with Few Data and the Training Sample Order," Pattern Recognition, Volume 61, January 2017, Pages 610-628

[7] Byoung Chul Ko," A Brief Review of Facial Emotion Recognition Based on Visual Information," Sensors (Basel). 2018 Feb; 18(2): 401. Published online 2018 Jan 30. Doi: 10.3390/s18020401, PMCID: PMC5856145m, PMID: 29385749

[8] Malika Arora, Munish Kumar, "Facial Emotion Recognition System Based on PCA and Gradient Features," National Academy Science Letters, December 2018, Volume 41, Issue 6, pp 365-368.

[9] Chérifa Z., Nasr Eddine B. (2018) Facial Expressions Recognition: Development and Application to HMI. In: Amine A., Mouhoub M., Ait Mohamed O., Djebbar B. (eds) Computational Intelligence and Its Applications. CIIA 2018. IFIP Advances in Information and Communication Technology, Vol 522. Springer, Cham.

[10] [10]. Deepak KumarJain, PouryaShamsolmoali ,ParamjitSehdev, "Extended Deep Neural Network for Facial Emotion Recognition," Pattern Recognition Letters, Volume 120, 1 April 2019, Pages 69-74.

[11] I. Lasri, A. R. Solh and M. E. Belkacemi, "Facial Emotion Recognition of Students using Convolutional Neural Network," 2019 Third International Conference on Intelligent Computing in Data Sciences (ICDS), Marrakech, Morocco, 2019, pp. 1-6, doi: 10.1109/ICDS47004.2019.8942386.

[12] A. John, A. MC, A. S. Ajayan, S. Sanoop and V. R. Kumar, "Real-Time Facial Emotion Recognition System With Improved Preprocessing and Feature Extraction," 2020 Third International Conference on Smart Systems and Inventive Technology (ICSSIT), Tirunelveli, India, 2020, pp. 1328-1333, doi: 10.1109/ICSSIT48917.2020.9214207

[13] Z. Zhang, M. Yi, J. Xu, R. Zhang and J. Shen, "Two-stage Recognition and Beyond for Compound Facial Emotion Recognition," 2020 15th IEEE International Conference on Automatic Face and Gesture Recognition (FG 2020), Buenos Aires, Argentina, 2020, pp. 900904, doi: 10.1109/FG47880.2020.00144.

[14] Tuncer, T., Dogan, S., Abdar, M. et al. A Novel Facial Image Recognition Method Based On Perceptual Hash Using Quintet Triple Binary Pattern. Multimed Tools Appl 79, 29573-29593 (2020). https://doi.org/10.1007/s11042-020-09439-8

[15] Chirra, V.R.R., Uyyala, S.R. \& Kolli, V.K.K. Virtual Facial Expression Recognition Using Deep CNN with Ensemble Learning. J Ambient Intell Human Comput (2021).

[16] Caroppo, A., Leone, A. \& Siciliano, P. Comparison Between Deep Learning Models and Traditional Machine Learning Approaches for Facial Expression Recognition in Ageing Adults. J. Comput. Sci. Technol. 35, 1127-1146 (2020). https://doi.org/10.1007/s11390-0209665-4

[17] Tcherkassof, A., Dupré, D. The Emotion-Facial Expression Link: Evidence from Human and Automatic Expression Recognition. Psychological Research (2020). https://doi.org/10.1007/s00426-020-01448-4

[18] Ismath Unnisa; Dr. Loganathan. R; "Human Facial Emotion Recognition using Adaptive Sigmoidal Transfer Function in Mlp Neural Network," International Journal of Engineering and Advanced Technology (IJEAT), 2019, Volume-9 Regular Issue-1, Scopus indexed, pp: 4103-4113.

[19] Ismath Unnisa; Dr. Loganathan. R; "PCA- Adaptive RBF Neural Network Hybridization for High Speed Face Recognition” in the “International Journal of Advanced Management Technology and Engineering Sciences” Volume 7, Issue 9, September-2017, http://ijamtes.org/VOL-7-ISSUE-7-2017/.

[20] The Yale Face Database http://vision.ucsd.edu/ 


\section{Appendix}

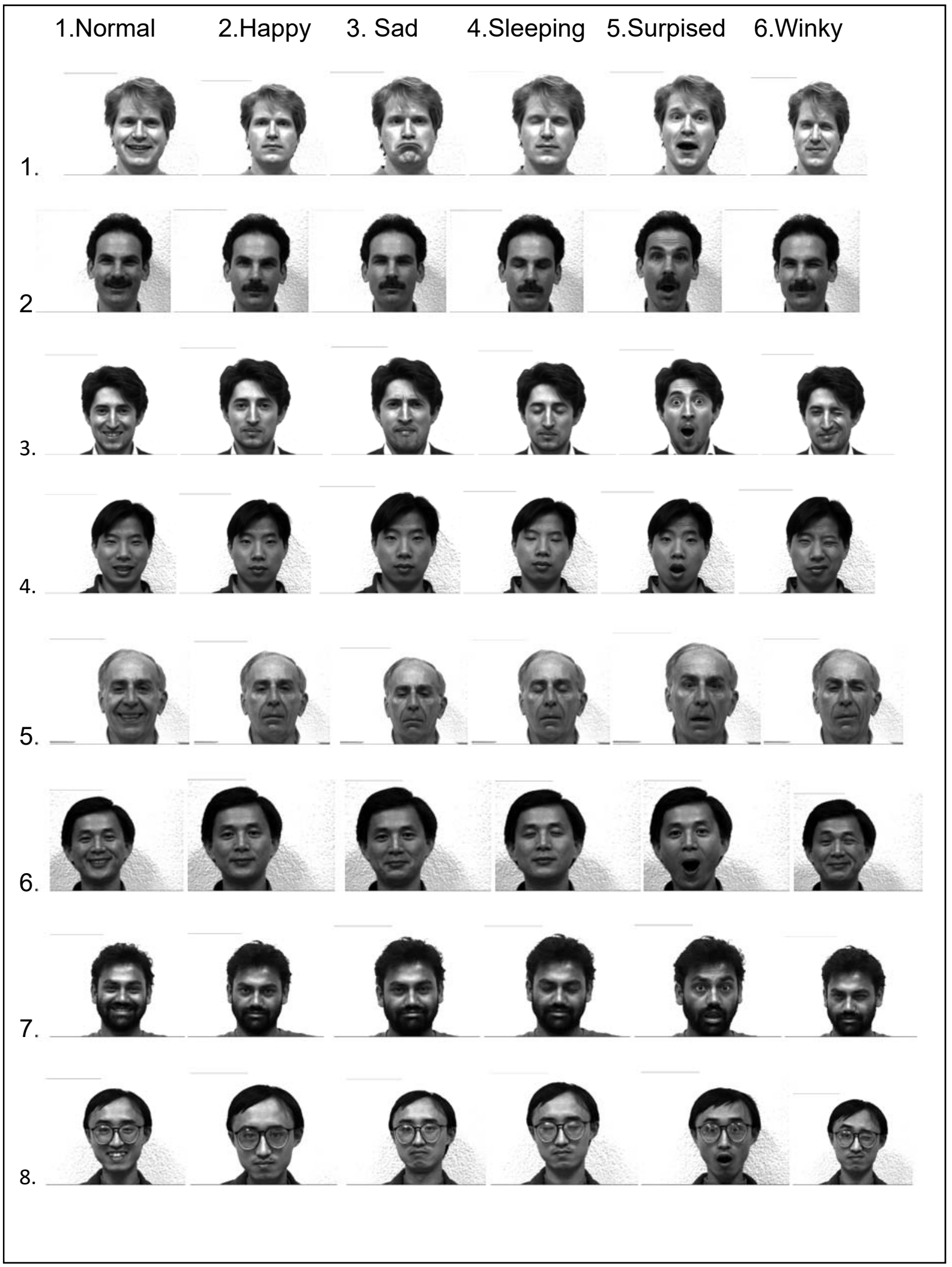




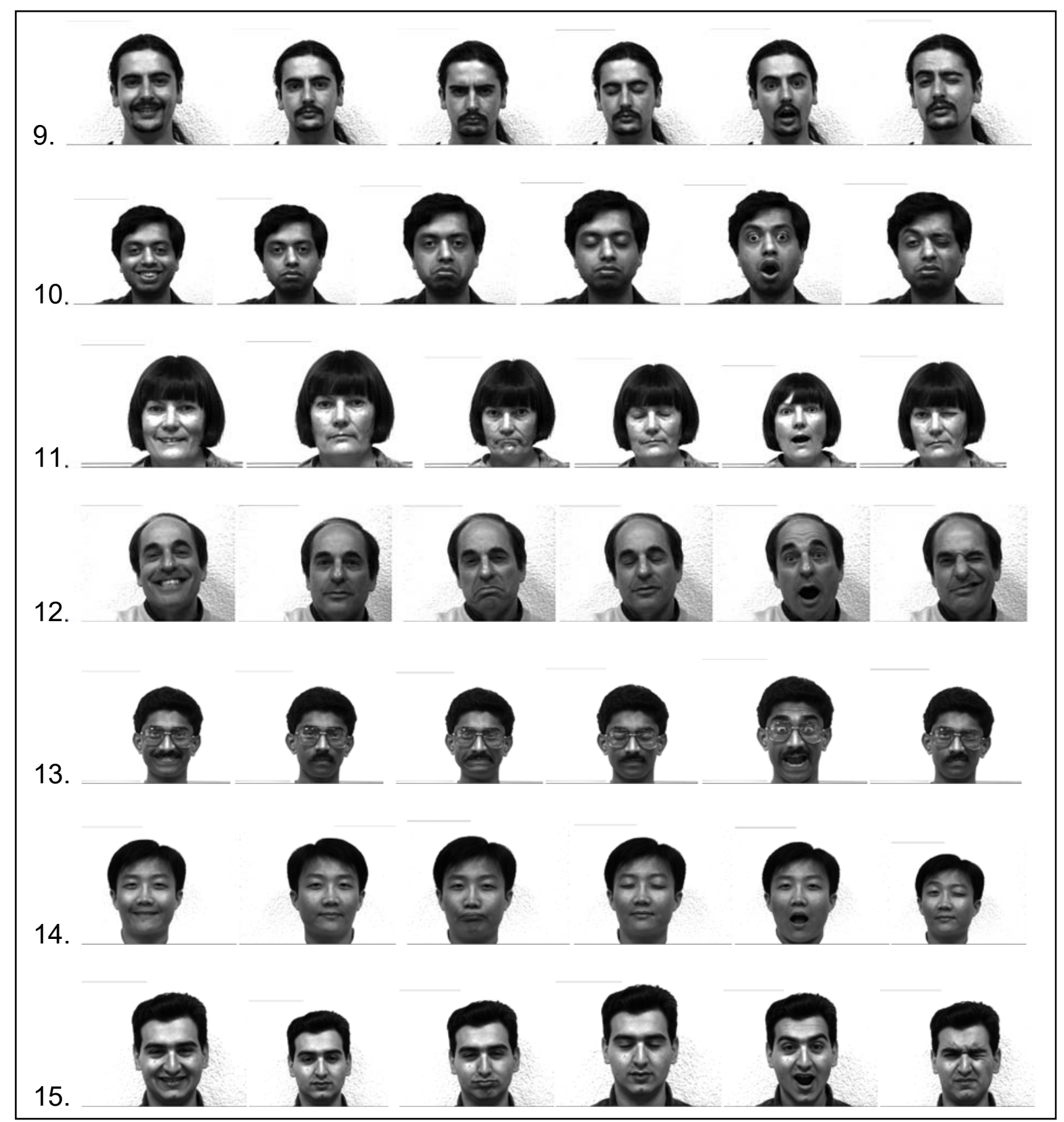

8. Authors Profile

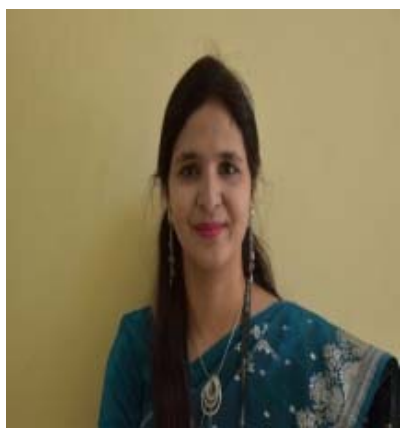

Mrs. Ismath Unnisa is a Research Scholar in Computer Science \& Engineering at HKBK College of Engineering, VTU, Belagavi with research title "Hybridization of Face and Associated Emotion Recognition using Evolved Neural Network." I have both teaching and research experience. I completed my M. Tech from The Oxford College of Engineering, Bangalore, in the year 2012. I have presented and published 11 papers at National and International Journals and also awarded second prize for the paper presented at M.S. Ramaiah Institute of Technology, Bangalore. My current interests include image processing and analysis, facial recognition, emotional recognition, computer vision and pattern recognition. 


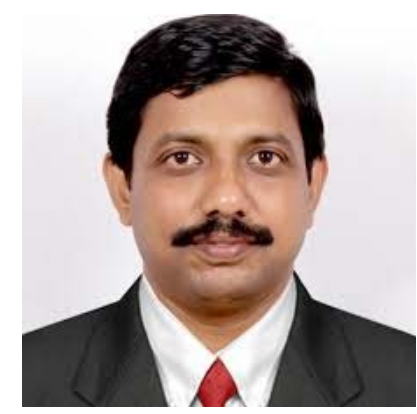

Dr. Loganathan $\mathbf{R}$ received a Ph.D degree in Computer Science \& Engineering from the Sathyabama University, Tamilnadu for the thesis entitled "A Novel Medical Image Compression Technique with Lossless Region of Interest using Adaptive Active Contour" in the year 2014. I have published more than 20 papers in journals and also presented more than 10 papers in National and International conferences. I am guiding 6 members in pursuing their Ph.D. I am having 25 Years of experience in teaching. I am working at HKBK College of Engineering since 1995, presently designated as Professor \& Head of Computer Science \& Engineering Department. I am also a member of professional bodies for ISTE and CSI. My current interests include image processing and analysis, facial recognition, emotional recognition, computer vision and pattern recognition. 